QUARTERLY OF APPLIED MATHEMATICS

VOLUME LXIII, NUMBER 1

MARCH 2005, PAGES 13-19

S $0033-569 X(05) 00946-2$

Article electronically published on January 19, 2005

\title{
INSTABILITY OF SOLUTIONS OF A SEMILINEAR HEAT EQUATION WITH A NEUMANN BOUNDARY CONDITION
}

\author{
BY \\ KENG DENG (Department of Mathematics, University of Louisiana at Lafayette, Lafayette, \\ Louisiana 70504) \\ AND \\ CHENG-LIN ZHAO (Department of Mathematics, University of Louisiana at Lafayette, Lafayette, \\ Louisiana 70504)
}

Abstract. A semilinear heat equation $u_{t}=u_{x x}+\varepsilon u^{p}, 0<x<1, \varepsilon, p>0$, subject to $u_{x}(0, t)=0, u_{x}(1, t)=-u^{-q}(1, t), q>0$ is studied. The set of stationary states is characterized, their instability is analyzed, and the large time behavior of positive solutions is discussed.

1. Introduction. In this paper, we consider the following initial-boundary value problem:

$$
\begin{array}{lll}
u_{t}=u_{x x}+\varepsilon u^{p} & 0<x<1, & t>0 \\
u_{x}(0, t)=0, u_{x}(1, t)=-u^{-q}(1, t) & & t>0 \\
u(x, 0)=u_{0}(x) & 0 \leq x \leq 1 .
\end{array}
$$

Here $\varepsilon, p, q>0$ and $u_{0}(x)$ is a positive function with $u_{0}^{\prime}(0)=0, u_{0}^{\prime}(1)=-u_{0}^{-q}(1)$. Problem (1.1) is a one-dimensional radially symmetric case for a model dealt with in 2. Physically, (1.1) can be treated as a heat conduction model that incorporates the effects of reaction and nonlinear outflux. Mathematically, (1.1) is a combination of the following two problems:

$$
\begin{array}{lll}
u_{t}=u_{x x}+\varepsilon u^{p} & 0<x<1, & t>0, \\
u_{x}(0, t)=u_{x}(1, t)=0 & & t>0, \\
u(x, 0)=u_{0}(x) & 0 \leq x \leq 1 &
\end{array}
$$

and

$$
\begin{array}{lll}
u_{t}=u_{x x} & 0<x<1, & t>0 \\
u_{x}(0, t)=0, u_{x}(1, t)=-u^{-q}(1, t) & & t>0 \\
u(x, 0)=u_{0}(x) & 0 \leq x \leq 1 .
\end{array}
$$

Received July 24, 2003.

2000 Mathematics Subject Classification. Primary 34B18, 35B05, 35B35, 35K60.

The work of the first author was supported in part by the National Science Foundation under grant DMS-0211412. 
Problems (1.2) and (1.3) have been intensively studied over the past few years. For details, see [1, 3, 4, 6] and the references cited therein.

In [2], we established criteria for finite time blow-up and quenching (the solution reaching zero), discussed blow-up and quenching sets, and obtained blow-up and quenching rates. In contrast to the results in [2], our main objectives here are to characterize the set of stationary states of (1.1), to present the instability property of these states, and to investigate the large time behavior of solutions of (1.1).

2. Stationary solutions. In this section, we study positive stationary solutions of problem (1.1), which satisfy the following nonlinear boundary value problem:

$$
\begin{aligned}
& v^{\prime \prime}(x)+\varepsilon v^{p}(x)=0, \quad 0<x<1, \\
& v^{\prime}(0)=0, \quad v^{\prime}(1)=-v^{-q}(1) .
\end{aligned}
$$

First, we note that because $v^{\prime \prime}(x)<0$ in $(0,1)$ and $v^{\prime}(0)=0, v(x)$ attains its maximum $M=v(0)$ and minimum $m=v(1)$. Moreover, $v(x)$ satisfies

$$
\frac{1}{2}\left(v^{\prime}(x)\right)^{2}+\frac{\varepsilon}{p+1}\left(v^{p+1}(x)-M^{p+1}\right)=0 .
$$

Integrating $(2.2)$ over $(0, x)$ then yields

$$
\int_{v(x)}^{M} \frac{d \tau}{\sqrt{M^{p+1}-\tau^{p+1}}}=\sqrt{\frac{2 \varepsilon}{p+1}} x .
$$

In particular, we have

$$
\int_{m}^{M} \frac{d \tau}{\sqrt{M^{p+1}-\tau^{p+1}}}=\sqrt{\frac{2 \varepsilon}{p+1}}
$$

Letting $\mu=m / M$, we introduce the function $\theta(\mu)=\sqrt{1-\mu^{p+1}}$. Since $\theta(\mu)$ is strictly decreasing in $(0,1)$, equation $(2.4)$ is equivalent to the following:

$$
M^{\frac{1-p}{2}} \int_{0}^{\theta} \frac{d \sigma}{\left(1-\sigma^{2}\right)^{\frac{p}{p+1}}}=\sqrt{\frac{(p+1) \varepsilon}{2}} \quad \theta \in(0,1) .
$$

On the other hand, in view of the boundary condition $v^{\prime}(1)=-v^{-q}(1),(2.2)$ gives the following relation:

$$
\frac{1}{2} m^{-2 q}+\frac{\varepsilon}{p+1}\left(m^{p+1}-M^{p+1}\right)=0,
$$

or equivalently,

$$
M=\left(\frac{p+1}{2 \varepsilon}\right)^{\frac{1}{p+2 q+1}} \theta^{-\frac{2}{p+2 q+1}}\left(1-\theta^{2}\right)^{-\frac{2 q}{(p+1)(p+2 q+1)}} .
$$

A combination of (2.5) and (2.6) then leads to

$$
\theta^{\gamma}\left(1-\theta^{2}\right)^{\frac{q \gamma}{p+1}} \int_{0}^{\theta} \frac{d \sigma}{\left(1-\sigma^{2}\right)^{\frac{p}{p+1}}}=C(\varepsilon, p, q),
$$

where $\gamma=(p-1) /(p+2 q+1)$ and $C(\varepsilon, p, q)=[(p+1) / 2]^{(p+q) /(p+2 q+1)} \varepsilon^{(q+1) /(p+2 q+1)}$. By virtue of (2.6), there exists a one-to-one correspondence between $M$ and $\theta$, and thus by (2.3) we conclude that the number of solutions of (2.1) is the same as that of (2.7). 
Therefore, in order to characterize the set of stationary solutions, we should focus our attention on (2.7). For convenience, let $G(\theta)=F^{\gamma}(\theta) I(\theta)$, where $F(\theta)=\theta\left(1-\theta^{2}\right)^{q /(p+1)}$ and $I(\theta)=\int_{0}^{\theta}\left(1-\sigma^{2}\right)^{-\frac{p}{p+1}} d \sigma$. Clearly, $G(0)=0$ and

$$
\lim _{\theta \rightarrow 1^{-}} G(\theta)= \begin{cases}\infty, & p<1, \\ \frac{\pi}{2}, & p=1, \\ 0, & p>1 .\end{cases}
$$

We then present the following two lemmas for $G^{\prime}(\theta)$.

Lemma 2.1. If $0<p \leq 1, G^{\prime}(\theta)>0$ in $(0,1)$.

Proof. Through a straightforward calculation, we find

$$
\begin{aligned}
G^{\prime}(\theta) & =F^{\gamma}(\theta)\left\{\frac{(1-p)\left[(p+2 q+1) \theta^{2}-(p+1)\right]}{(p+1)(p+2 q+1) \theta\left(1-\theta^{2}\right)} I(\theta)+\left(1-\theta^{2}\right)^{-\frac{p}{p+1}}\right\} \\
& =F^{\gamma}(\theta) H(\theta) .
\end{aligned}
$$

If $0<p<1$, clearly, $G^{\prime}(\theta)>0$ for $\theta \geq \sqrt{(p+1) /(p+2 q+1)}$. For $0<\theta<$ $\sqrt{(p+1) /(p+2 q+1)}$, since $I(\theta) \leq \theta\left(1-\theta^{2}\right)^{-p /(p+1)}$, we have

$$
\begin{aligned}
H(\theta) & \geq\left\{\frac{(1-p)\left[(p+2 q+1) \theta^{2}-(p+1)\right]}{(p+1)(p+2 q+1)\left(1-\theta^{2}\right)}+1\right\}\left(1-\theta^{2}\right)^{-\frac{p}{p+1}} \\
& =\frac{2\left(1-\theta^{2}\right)^{-\frac{2 p+1}{p+1}}}{(p+1)(p+2 q+1)}\left[(p+1)(p+q)-p(p+2 q+1) \theta^{2}\right]>0 .
\end{aligned}
$$

If $p=1, G^{\prime}(\theta)=\left(1-\theta^{2}\right)^{-p /(p+1)}>0$.

Lemma 2.2. If $p>1$, there exists a $\hat{\theta} \in(0,1)$ such that $G^{\prime}(\theta)>0$ for $0<\theta<\hat{\theta}$ and $G^{\prime}(\theta)<0$ for $\hat{\theta}<\theta<1$.

Proof. Let $\alpha=p+2 q+1, \beta=p+1$, and $\eta=p-1$. Then $\alpha, \beta, \eta>0$ and $H(\theta)$ in (2.8) takes the form

$$
H(\theta)=\frac{\eta\left(\beta-\alpha \theta^{2}\right)}{\alpha \beta \theta\left(1-\theta^{2}\right)} I(\theta)+\left(1-\theta^{2}\right)^{\frac{1}{\beta}-1} .
$$

It follows from (2.9) that $H(\theta)>0$ for $0<\theta \leq \sqrt{\beta / \alpha}$, while for $\sqrt{\beta / \alpha}<\theta<1, H(\theta)$ has the same number of zeros as that of the function $A(\theta)$, given by

$$
A(\theta)=\frac{\alpha \beta \theta\left(1-\theta^{2}\right)^{\frac{1}{\beta}}}{\eta\left(\alpha \theta^{2}-\beta\right)}-I(\theta), \quad \sqrt{\frac{\beta}{\alpha}}<\theta<1 .
$$

Differentiation of (2.10) gives

$$
A^{\prime}(\theta)=\frac{\alpha \beta\left(1-\theta^{2}\right)^{\frac{1}{\beta}-1}}{\eta\left(\alpha \theta^{2}-\beta\right)^{2}} B\left(\theta^{2}\right), \quad \sqrt{\frac{\beta}{\alpha}}<\theta<1,
$$

where $B(t)=2[(p-q) t-(p+q)(p+1) /(p+2 q+1)]$ for $t \in(\beta / \alpha, 1)$. If $p \leq q, B(t)<0$, and hence $A^{\prime}(\theta)<0$ in $(\sqrt{\beta / \alpha}, 1)$, which implies that $G^{\prime}(\theta)$ has at most one zero in $(0,1)$. Since $\lim _{\theta \rightarrow \sqrt{\beta / \alpha}^{+}} A(\theta)=\infty$ and $A(1)<0, G^{\prime}(\theta)$ has exactly one zero in $(0,1)$. If $p>q$, letting $\theta_{0}^{2}=(p+q)(p+1) /(p+2 q+1)(p-q)$, it is easily seen that $A^{\prime}\left(\theta_{0}\right)=0$ and $A^{\prime \prime}\left(\theta_{0}\right)>0$. Thus $A(\theta)$ attains its minimum at $\theta_{0}$, which again implies that $G^{\prime}(\theta)$ has exactly one zero in $(0,1)$. 
From the above monotonicity properties and the behavior of $G(\theta)$ near the two endpoints, we obtain the following result.

TheOREm 2.3. If $0<p<1$, problem (2.1) has one positive solution for any $\varepsilon>0$. If $p=1$, (2.1) has one positive solution for $\varepsilon \leq \pi^{2} / 4$ and no solutions for $\varepsilon>\pi^{2} / 4$. If $p>1$, there exists a critical number $\varepsilon_{0}(p, q)$ such that $(2.1)$ has two positive solutions for $0<\varepsilon<\varepsilon_{0}$, one positive solution for $\varepsilon=\varepsilon_{0}$, and no solutions for $\varepsilon>\varepsilon_{0}$.

Let $v(x)$, or sometimes $v(x, \varepsilon)$, denote the stationary solutions of (1.1). Since $v(x, \varepsilon)$ depends on $v(0, \varepsilon)$ continuously and $v(0, \varepsilon)$ is a continuous function of $\varepsilon, v(x, \varepsilon)$ is a continuous function of $\varepsilon$.

For $p>1$ and $0<\varepsilon<\varepsilon_{0}$, let $v_{1}(x)$ and $v_{2}(x)$ denote the stationary solutions with $\mu_{1}$ and $\mu_{2}\left(\mu_{1}<\mu_{2}\right)$, respectively. We present the following result, which contrasts sharply with that in [5].

THEOREM 2.4. If $p>1$ and $0<\varepsilon<\varepsilon_{0}$, the two solutions $v_{1}(x)$ and $v_{2}(x)$ are not ordered.

Proof. Let $M_{i}$ and $m_{i}$ denote $v_{i}(0)$ and $v_{i}(1)(i=1,2)$, respectively. By $(2.5)$ we have

$$
M_{1}^{\frac{1-p}{2}} \int_{0}^{\theta_{1}} \frac{d \sigma}{\left(1-\sigma^{2}\right)^{\frac{p}{p+1}}}=M_{2}^{\frac{1-p}{2}} \int_{0}^{\theta_{2}} \frac{d \sigma}{\left(1-\sigma^{2}\right)^{\frac{p}{p+1}}} .
$$

Since $\mu_{1}<\mu_{2}, \theta_{1}>\theta_{2}$, and thus it follows from (2.11) that $M_{1}^{\frac{1-p}{2}}<M_{2}^{\frac{1-p}{2}}$, i.e., $M_{1}>M_{2}$. On the other hand, by (2.6) and the transformation $\mu=\left(1-\theta^{2}\right)^{1 /(p+1)}$, we find

$$
m=\left(\frac{p+1}{2 \varepsilon}\right)^{\frac{1}{p+2 q+1}} \theta^{-\frac{2}{p+2 q+1}}\left(1-\theta^{2}\right)^{\frac{1}{p+2 q+1}},
$$

which shows $m_{1}<m_{2}$.

3. Instability and large time behavior. In this section, we first establish the instability result. Without making any confusion, sometimes we shall write the solution of $(1.1)$ as $u(x, t, \varepsilon)$ or $u\left(x, t ; u_{0}\right)$ with $u_{0}$ being the initial datum.

We begin by formulating the precise notion of stability.

Definition. A stationary solution, $v(x)$, of (1.1) is stable if for any given $\rho>0$ there exists a $\sigma>0$ such that if $u(x, t)$ is a positive solution of (1.1) with $|u(x, 0)-v(x)|<\sigma$ on $[0,1]$, then $\|u(\cdot, t)-v(\cdot)\|_{\infty}<\rho$ for all $t \geq 0$, and $\lim _{t \rightarrow \infty} u(x, t)=v(x)$ for each $x \in[0,1]$.

THEOREM 3.1. Every stationary solution of (1.1) is unstable.

Proof. Let $\mu(\varepsilon)=v(1, \varepsilon) / v(0, \varepsilon)$. We consider two cases.

Case 1. $\mu^{\prime}(\varepsilon)>0$. This case corresponds to $p>1$ and $\theta>\hat{\theta}$, i.e., $\mu(\varepsilon)=\mu_{1}(\varepsilon)$, where $\hat{\theta}$ is the critical point of $G(\theta)$ in Lemma 2.2. Making use of (2.4), we have

$$
M^{\frac{1-p}{2}} \int_{\mu}^{1} \frac{d \zeta}{\sqrt{1-\zeta^{p+1}}}=\sqrt{\frac{2 \varepsilon}{p+1}} .
$$


For $0<\varepsilon_{1}<\varepsilon_{2}<\varepsilon_{0}$, we then find

$$
M_{1}^{\frac{1-p}{2}}\left(\varepsilon_{1}\right) \int_{\mu_{1}\left(\varepsilon_{1}\right)}^{1} \frac{d \zeta}{\sqrt{1-\zeta^{p+1}}}<M_{1}^{\frac{1-p}{2}}\left(\varepsilon_{2}\right) \int_{\mu_{1}\left(\varepsilon_{2}\right)}^{1} \frac{d \zeta}{\sqrt{1-\zeta^{p+1}}} .
$$

Since $\mu_{1}\left(\varepsilon_{1}\right)<\mu_{1}\left(\varepsilon_{2}\right)$, by $(3.2) M_{1}^{\frac{1-p}{2}}\left(\varepsilon_{1}\right)<M_{1}^{\frac{1-p}{2}}\left(\varepsilon_{2}\right)$, i.e., $M_{1}\left(\varepsilon_{1}\right)>M_{1}\left(\varepsilon_{2}\right)$. Thus we know that $v_{1}\left(x, \varepsilon_{1}\right)>v_{1}\left(x, \varepsilon_{2}\right)$ in a subinterval $\left[0, x_{0}\right]\left(0<x_{0}<1\right)$. Let $u\left(x, t, \varepsilon_{2}\right)$ be a solution of $(1.1)$ with $u_{0}(x)=v_{1}\left(x, \varepsilon_{1}\right)$. Then in $(0,1)$, we find that

$$
u_{0}^{\prime \prime}+\varepsilon_{2} u_{0}^{p}=v_{1}^{\prime \prime}\left(x, \varepsilon_{1}\right)+\varepsilon_{2} v_{1}^{p}\left(x, \varepsilon_{1}\right)>v_{1}^{\prime \prime}\left(x, \varepsilon_{1}\right)+\varepsilon_{1} v_{1}^{p}\left(x, \varepsilon_{1}\right)=0 .
$$

Hence $u_{t} \geq 0$ for $t>0$. Consequently, $u\left(x, t, \varepsilon_{2}\right)$ is increasing in $t$, which indicates that $v_{1}\left(x, \varepsilon_{2}\right)$ is unstable. Similarly, it can be shown that with $\varepsilon_{1}>\varepsilon_{2}, v_{1}\left(x, \varepsilon_{2}\right)$ is unstable.

Case 2. $\mu^{\prime}(\varepsilon)<0$. This case corresponds to $0<p \leq 1$ or $p>1$ and $\theta<\hat{\theta}$, i.e., $\mu(\varepsilon)=\mu_{2}(\varepsilon)$. Since $\theta^{\prime}(\mu)<0, \theta^{\prime}(\varepsilon)>0$. In view of (2.12), for $0<\varepsilon_{1}<\varepsilon_{2}<\infty$, we have $m\left(\varepsilon_{1}\right)>m\left(\varepsilon_{2}\right)$. Thus we know that $v\left(x, \varepsilon_{1}\right)>v\left(x, \varepsilon_{2}\right)$ in a subinterval $\left[x_{1}, 1\right](0<$ $\left.x_{1}<1\right)$. Proceeding analogously, one can see that $v\left(x, \varepsilon_{2}\right)$ is unstable.

Finally, we note that in a similar manner, we can show that for $p>1, v\left(x, \varepsilon_{0}\right)$ is also unstable.

To discuss the large time behavior of solutions of (1.1), we need the following two lemmas, whose proofs are identical to those in [2] and hence are omitted.

Lemma 3.2. Suppose that $u_{0}^{\prime \prime}(x)+\varepsilon u_{0}^{p}(x) \geq 0$ for $0<x<1$ and $p>1$. Then the solution of (1.1) blows up in finite time.

Lemma 3.3. Suppose that $u_{0}^{\prime \prime}(x)+\varepsilon u_{0}^{p}(x) \leq 0$ for $0<x<1$ and $p>0$. Then the solution of (1.1) quenches in finite time.

We are now in a position to establish the following result.

Theorem 3.4. (I) Let $0<p<1$. For any $\varepsilon>0$, if $u_{0}(x) \leq v(x, \varepsilon)$, $u$ quenches in finite time, whereas $u$ exists globally and $\lim _{t \rightarrow \infty} u(x, t)=\infty$ if $u_{0}(x) \geq v(x, \varepsilon)$.

(II) Let $p=1$.

(a) For $\varepsilon<\frac{\pi^{2}}{4}$, if $u_{0}(x) \leq v(x, \varepsilon), u$ quenches in finite time, while $u$ is global and $\lim _{t \rightarrow \infty} u(x, t)=\infty$ if $u_{0}(x) \geq v(x, \varepsilon)$.

(b) For $\varepsilon \geq \frac{\pi^{2}}{4}$, if $u_{0}(x) \geq v\left(x, \frac{\pi^{2}}{4}\right), u$ exists for all $t>0$ and $\lim _{t \rightarrow \infty} u(x, t)=\infty$.

(III) Let $p>1$.

(a) For $\varepsilon<\varepsilon_{0}$, if $u_{0}(x) \leq v_{i}(x, \varepsilon)(i=1$ or $i=2), u$ quenches in finite time, whereas $u$ blows up in finite time if $u_{0}(x) \geq v_{i}(x, \varepsilon)(i=1$ or $i=2)$.

(b) For $\varepsilon \geq \varepsilon_{0}$, if $u_{0}(x) \geq v\left(x, \varepsilon_{0}\right)$, $u$ blows up in finite time.

Proof. (I) If $u_{0}(x) \leq v(x, \varepsilon)$, by the comparison principle, $u(x, t)<v(x, \varepsilon)$ for any $t>0$. Thus without loss of generality, we may assume $u_{0}(x)<v(x, \varepsilon)$. We then choose $\bar{u}_{0}(x)=v(x, \delta)$ with $\delta>\varepsilon$ such that $u_{0} \leq \bar{u}_{0}$ in $[0,1]$. By comparison, $u\left(x, t ; u_{0}\right) \leq$ $u\left(x, t ; \bar{u}_{0}\right)$. Moreover,

$$
\bar{u}_{0}^{\prime \prime}+\varepsilon \bar{u}_{0}^{p}=v^{\prime \prime}(x, \delta)+\varepsilon v^{p}(x, \delta)<v^{\prime \prime}(x, \delta)+\delta v^{p}(x, \delta)=0 .
$$

By Lemma $3.3, u\left(x, t ; \bar{u}_{0}\right)$ quenches in finite time, and so does $u\left(x, t ; u_{0}\right)$. 
If $u_{0}(x) \geq v(x, \varepsilon)$, we select $\underline{u}_{0}(x)=v(x, \delta)$ with $\delta<\varepsilon$ such that $u_{0} \geq \underline{u}_{0}$ in $[0,1]$. Since $\underline{u}_{0}^{\prime \prime}+\varepsilon \underline{u}_{0}^{p}>0, u\left(x, t ; \underline{u}_{0}\right)$ is monotonically increasing. On the other hand, it is easy to verify that $w(x, t)=M e^{\varepsilon t}$ is an upper solution of (1.1), where $M=\max \left\{1, \max _{0 \leq x \leq 1} u_{0}(x)\right\}$.

Thus by comparison, $u\left(x, t ; \underline{u}_{0}\right) \leq u\left(x, t ; u_{0}\right) \leq w(x, t)$, and it follows that $u\left(x, t ; u_{0}\right)$ is global. If $\lim _{t \rightarrow \infty} u\left(x, t ; u_{0}\right)<\infty$, recalling the result of [7], $u$ must converge to its steady state. However, $u\left(1, t ; u_{0}\right) \geq u\left(1, t ; \underline{u}_{0}\right) \geq v(1, \delta)>v(1, \varepsilon)$, which means that $\lim _{t \rightarrow \infty} u\left(x, t ; u_{0}\right)=\infty$.

(II) The proof is similar to that for (I) and hence is omitted.

(III) (a) Proof for the case $u_{0}(x) \leq v_{i}(x, \varepsilon)$ is the same as that in (I). If $u_{0}(x) \geq v_{i}(x, \varepsilon)$, we choose $\underline{u}_{0}(x)=v_{i}(x, \delta)$ with $\delta<\varepsilon$ such that $u_{0} \geq \underline{u}_{0}$ in $[0,1]$. Since $\underline{u}_{0}^{\prime \prime}+\varepsilon \underline{u}_{0}^{p}>0$, by Lemma $3.2, u\left(x, t ; \underline{u}_{0}\right)$ blows up in finite time, and so does $u\left(x, t ; u_{0}\right)$.

(b) We can argue in a similar manner, and hence the proof is omitted.

REMARK 1. If $0<p \leq 1$, for any initial data $u_{0}(x)$, by comparison, the solution cannot blow up in finite time. If $p>1$, when $0<u_{0}(x)<1$, the solution should quench as $q$ increases beyond some critical value. The reason for this is that in view of (2.12), as $q$ increases, problem (1.1) will have a stationary solution $v(x) \geq u_{0}(x)$. However, the situation could just as reasonably go the other way if $u_{0}(x)$ is very large at $x=1$. In such case, for any $q>0$, problem (1.1) is initially close to problem (1.2), and finite time blow-up is to be expected.

REMARK 2. Since the flux condition at $x=1$ can be rewritten as $\left(u^{q+1}\right)_{x}=-(q+1)$, and this allows a weak formulation of the problem that makes sense even if a solution vanishes, a natural question arises: Is it possible to extend solutions beyond quenching? It appears that the same thing occurs with the porous medium equation, wherein the solution $u$ is nondifferentiable whenever it vanishes, yet $u^{m}$ is differentiable. Subsequently, solutions develop sharp interfaces between where they vanish and where they are positive. Once it is possible to extend solutions beyond quenching, one could also ask whether a solution would possibly blow up in finite time even after quenching has occurred. Such questions obviously deserve our future consideration.

Acknowledgement. The authors would like to thank the referee for several helpful comments.

\section{REFERENCES}

[1] K. Deng and M. Xu, Quenching for a nonlinear diffusion equation with a singular boundary condition, Z. Angew. Math. Phys. 50 (1999), 574-584. MR1709705 (2000e:35110)

[2] K. Deng and C.-L. Zhao, Blow-up versus quenching, Comm. Appl. Anal. 7 (2003), 87-100. MR.1954906 (2003j:35170)

[3] M. Fila and H.A. Levine, Quenching on the boundary, Nonlinear Anal. TMA 21 (1993), 795-802. MR:1246508 (95b:35028)

[4] A. Friedman and B. Mcleod, Blow-up of positive solutions of semilinear heat equations, Indiana Univ. Math. J. 34 (1985), 425-447. MR0783924|(86j:35089)

[5] H.A. Levine, Quenching, nonquenching, and beyond quenching for solutions of some parabolic equations, Ann. Mat. Pura Appl. 155 (1989), 243-260. MR1042837 (91m:35028)

[6] C.V. Pao, Nonlinear Parabolic and Elliptic Equations, Plenum Press, New York, 1992. MR1212084 (94c:35002) 
[7] T.I. Zelenyak, Stabilisation of solutions of boundary value problems for a second-order equation with one space variable, Differential Equations 4 (1968), 17-22. 